Marquette University

e-Publications@Marquette

Biomedical Sciences Faculty Research and

Publications

Biomedical Sciences, Department of

8-1-2020

\title{
Reduced VIP Expression Affects Circadian Clock Function in VIP- IRES-CRE Mice (JAX 010908)
}

Deborah A.M. Joye

Marquette University

Kayla Rohr

Marquette University

Danielle Keller

Marquette University

Thomas Inda

Marquette University

Adam Telega

Marquette University

See next page for additional authors

Follow this and additional works at: https://epublications.marquette.edu/biomedsci_fac

Part of the Neurosciences Commons

\section{Recommended Citation}

Joye, Deborah A.M.; Rohr, Kayla; Keller, Danielle; Inda, Thomas; Telega, Adam; Pancholi, Harshida; Carmonia-Alcocer, Vania; and Evans, Jennifer A., "Reduced VIP Expression Affects Circadian Clock Function in VIP-IRES-CRE Mice (JAX 010908)" (2020). Biomedical Sciences Faculty Research and Publications. 195.

https://epublications.marquette.edu/biomedsci_fac/195 


\section{Authors}

Deborah A.M. Joye, Kayla Rohr, Danielle Keller, Thomas Inda, Adam Telega, Harshida Pancholi, Vania Carmonia-Alcocer, and Jennifer A. Evans 
Marquette University

e-Publications@Marquette

\section{Biomedical Sciences Faculty Research and Publications/College of Health Sciences}

This paper is NOT THE PUBLISHED VERSION; but the author's final, peer-reviewed manuscript. The published version may be accessed by following the link in the citation below.

Journal of Biological Rhythms, Vol. 35, No. 4 (August 1, 2020): 340-352. DOI. This article is (C) SAGE Publications and permission has been granted for this version to appear in e-Publications@Marquette. SAGE Publications does not grant permission for this article to be further copied/distributed or hosted elsewhere without the express permission from SAGE Publications.

\section{Reduced VIP Expression Affects Circadian Clock Function in VIP-IRES-CRE Mice (JAX 010908)}

Deborah A. M. Joye

Department of Biomedical Sciences, Marquette University, Milwaukee, Wisconsin

Kayla E. Rohr

Department of Biomedical Sciences, Marquette University, Milwaukee, Wisconsin Danielle Keller Department of Biomedical Sciences, Marquette University, Milwaukee, Wisconsin Thomas Inda

Department of Biomedical Sciences, Marquette University, Milwaukee, Wisconsin Adam Telega Department of Biomedical Sciences, Marquette University, Milwaukee, Wisconsin Harshida Pancholi

Department of Biomedical Sciences, Marquette University, Milwaukee, Wisconsin 


\title{
Vania Carmona-Alcocer
}

Department of Biomedical Sciences, Marquette University, Milwaukee, Wisconsin

Jennifer A. Evans

Department of Biomedical Sciences, Marquette University, Milwaukee, Wisconsin

\begin{abstract}
Circadian rhythms are programmed by the suprachiasmatic nucleus (SCN), which relies on neuropeptide signaling to maintain daily timekeeping. Vasoactive intestinal polypeptide (VIP) is critical for SCN function, but the precise role of VIP neurons in SCN circuits is not fully established. To interrogate their contribution to SCN circuits, VIP neurons can be manipulated specifically using the DNA-editing enzyme Cre recombinase. Although the Cre transgene is assumed to be inert by itself, we find that VIP expression is reduced in both heterozygous and homozygous adult VIP-IRES-Cre mice (JAX 010908). Compared with wild-type mice, homozygous VIP-Cre mice display faster reentrainment and shorter free-running period but do not become arrhythmic in constant darkness. Consistent with this phenotype, homozygous VIP-Cre mice display intact SCN PER2::LUC rhythms, albeit with altered period and network organization. We present evidence that the ability to sustain molecular rhythms in the VIP-Cre SCN is not due to residual VIP signaling; rather, arginine vasopressin signaling helps to sustain SCN function at both intracellular and intercellular levels in this model. This work establishes that the VIP-IRES-Cre transgene interferes with VIP expression but that loss of VIP can be mitigated by other neuropeptide signals to help sustain SCN function. Our findings have implications for studies employing this transgenic model and provide novel insight into neuropeptide signals that sustain daily timekeeping in the master clock.
\end{abstract}

\section{Keywords}

VIP-IRES-CRE mice (JAX 010908), circadian, vasoactive intestinal polypeptide, suprachiasmatic nucleus, cre transgene, neuropeptides, arginine vasopressin

Daily rhythms in behavior and physiology are programmed by the circadian system, which is a hierarchical collection of clocks coordinated by a master pacemaker in the anterior hypothalamus (Moore, 2013). At the molecular level, circadian rhythms are generated by transcriptional-translational feedback loops that regulate the daily expression of clock genes and their protein products (Buhr and Takahashi, 2013; Partch et al., 2014). At its core, this involves a 24-h negative feedback loop in which CLOCK-BMAL1 activates Period (Per1, Per2) and Cryptochrome (Cry1, Cry2) genes, which are translated into protein products that repress their own transcription (Buhr and Takahashi, 2013). This molecular mechanism operates in nearly every cell to regulate cellular physiology in a tissue-specific manner (Zhang et al., 2014). At the system level, these numerous tissue clocks rely on signals from the master clock in the suprachiasmatic nucleus (SCN) to maintain internal synchrony and entrainment with the 24-h environment. Like other cells of the body, SCN cells possess an intrinsic molecular clock; however, intercellular coupling is necessary for master clock function (Evans, 2016; Hastings et al., 2018). Thus, 
to understand the daily control of behavior and physiology, it is critical to delineate the circuits and signals by which SCN neurons communicate with one another.

The SCN neuropeptide vasoactive intestinal polypeptide (VIP) plays an important role in sustaining circadian rhythms at the behavioral, cellular, and molecular levels (Evans, 2016; Hastings et al., 2018; Vosko et al., 2007). Mice lacking VIP or its cognate receptor VPAC2 display locomotor rhythms that are arrhythmic or low amplitude with a short period in constant darkness (Aton et al., 2005; Ciarleglio et al., 2009; Colwell et al., 2003; Harmar et al., 2002; Power et al., 2010). In addition, VIP-deficient mice also display aberrant responses to photic stimuli, including accelerated recovery from simulated jetlag (Colwell et al., 2003; Harmar et al., 2002; Hughes et al., 2015; Hughes et al., 2004; Hughes and Piggins, 2008; Lucassen et al., 2012). Consistent with these behavioral deficits, SCN molecular and electrical rhythms are compromised in Vip ${ }^{-/}$and Vipr2/- knockout mice (Cutler et al., 2003; Harmar et al., 2002) because of deficits at the intracellular and intercellular levels (Aton et al., 2005; Brown et al., 2005; Ciarleglio et al., 2009; Maywood et al., 2006). Although recognized as an important neuropeptide, the precise role of VIP remains unclear since other SCN peptides are also altered in Vip ${ }^{-/}$and Vipr2\%- knockout mice (Bedont et al., 2018; Harmar et al., 2002). Thus, a deeper understanding of VIP signaling can be gained by genetic approaches employing targeted and conditional mutagenesis. A commonly used method for conditional mutagenesis is the Cre/loxP system, in which expression of the DNA-editing enzyme Cre recombinase is targeted to specific cellular populations (Matthaei, 2007). Technical advances have led to widespread use of this technique and refinements designed to mitigate off-targets effects (e.g., insertion of the IRES sequence). However, it has been noted that the transgene can interfere with gene expression even in the most wellconstructed models (Cheng et al., 2019; Hoffmann et al., 2019; Taniguchi et al., 2011; Viollet et al., 2017).

Given the increased use of the VIP-Cre mouse model in circadian studies, we examined potential effects of the transgene on both VIP expression and SCN clock function. One advantage to examining this particular genetic model is that the phenotypes and consequences of VIP deficiency are well established. In agreement with a recent report (Cheng et al., 2019), we find that VIP expression is reduced by the VIP-IRES-Cre transgene in adult heterozygous VIP-Cre mice (Vip ${ }^{\text {cre/+}}$, JAX 010908). Further, we demonstrate that VIP is decreased at an earlier age and in a more pronounced manner in homozygous VIP-Cre mice (Vip ${ }^{\text {cre/cre }}$ ). Functional knockdown of VIP is supported by findings that homozygous VIP-Cre mice display circadian phenotypes previously reported for VIP knockout mice. Surprisingly, despite almost complete loss of VIP in Vip cre/cre mice, arrhythmicity does not emerge at either the behavioral or SCN levels, suggesting that clock function is sustained by non-VIP signals in this model. Consistent with this hypothesis, inhibition of AVP signaling compromises PER2::LUC rhythms in the Vip ${ }^{\text {cre/cre }} \mathrm{SCN}$ more than in wild-type SCN. These data highlight not only an underappreciated caveat to working with this VIP-IRES-Cre mouse but also that the acquired deficiency in this model may provide new insight into the role of non-VIP signaling in sustaining SCN circuit function. 


\section{Methods and Materials}

\section{Mice and Husbandry Conditions}

Mice were bred and raised under a 24-h light-dark cycle with $12 \mathrm{~h}$ of light and $12 \mathrm{~h}$ of darkness (LD12:12; lights-off at $1800 \mathrm{~h} \mathrm{CST}$, defined as zeitgeber time 12 [ZT12]). Throughout life, ambient temperature was maintained at $22{ }^{\circ} \mathrm{C} \pm 2{ }^{\circ} \mathrm{C}$, and mice had ad libitum access to water and food (Teklad Rodent Diet 8604). Homozygous founder VIP-IRES-Cre ${ }^{+/+}$mice were obtained from Jackson Laboratory (Vip ${ }^{<\mathrm{tm} 1(\mathrm{cre}) Z \mathrm{jh}>} / \mathrm{J}$; stock No. 010908) and bred to PERIOD2::LUCIFERASE mice (Yoo et al., 2004) for at least 4 generations to produce homozygous VIP-Cre mice (Vip cre/cre), heterozygous VIP-Cre mice $\left(\right.$ Vip $\left.^{\text {cre/+ }}\right)$, and VIP wild-type mice $\left(\right.$ Vip $\left.^{+/+}\right)$. Mice used for behavior and immunohistochemistry were heterozygous for PER2::LUC, but those used for bioluminescence assays were homozygous for PER2::LUC. At weaning, mice were group housed in cages without running wheels. All procedures were conducted according to the National Institutes of Health Guide for the Care and Use of Animals and were approved by the Institutional Animal Care and Use Committee at Marquette University.

\section{Immunohistochemistry}

To test whether the VIP-IRES-Cre transgene interferes with neuropeptide expression, SCN were collected from male and female $\mathrm{Vip}^{+/+}, \mathrm{Vip}^{\mathrm{cre} /+}$, and Vip ${ }^{\text {cre/cre }}$ mice. Mice were deeply anesthetized with isoflurane and then sacrificed by cervical dislocation. Brains were collected on postnatal day 10 (P10, $n=7-8 /$ genotype) at ZT12, a time of day when VIP is expected to be elevated (Dardente et al., 2004; Isobe and Muramatsu, 1995). In adult mice (23-25 weeks old, $n=4$ /genotype), brains were collected $48 \mathrm{~h}$ after $1 \mu \mathrm{L}$ colchicine injection into the third ventricle $(0.5 \mu \mathrm{L} / \mathrm{min})$ to assess total peptide expression over the circadian cycle. After collection, brains were fixed in $4 \%$ paraformaldehyde, cryoprotected in $20 \%$ sucrose for 4 days, and then sectioned with a cryostat in the coronal plane. Freefloating SCN slices $(40 \mu \mathrm{m})$ were processed to detect VIP- and AVP-immunoreactivity, as described in Bedont et al. (2018). Briefly, slices were rinsed in phosphate-buffered saline (PBS), incubated for 48 $\mathrm{h}$ in primary antibodies (guinea pig-anti-AVP, 1:1000, Peninsula, cat No. T5048; rabbit-anti-VIP, 1:500, Peninsula, cat No. T4246), rinsed again in PBS, incubated for $2 \mathrm{~h}$ in secondary antibodies (donkey-antiguinea pig Alexa 594, 1:500, Jackson, cat No. 706-585-148; donkey-anti-rabbit Alexa 488, 1:500, Jackson, cat. No. 711-545-152), then rinsed a final time in PBS before being mounted with Prolong AntiFade medium + DAPI onto microscope slides and coverslipped. SCN slices were imaged with a Nikon 80i microscope fitted with a Retiga 2000R digital camera (QImaging, Surrey, BC, Canada) connected to a computer running NIS Elements-D software (Nikon Instruments, Melville NY, USA). Using ImageJ software, free-form regions of interest (ROIs) were drawn around the SCN borders using a DAPI staining as a guide. Within the SCN ROI, circular ROIs of standard size were placed on ventral and dorsomedial subregions to obtain average optical density measures of VIP and AVP levels, respectively (values corrected for background). For cell counting, the ventral SCN ROI was superimposed onto the thresholded images of VIP or DAPI and then submitted to the Analyze Particles function in ImageJ. For both optical density and cell count measures, values were normalized to wild type.

\section{Behavioral Analyses}

To evaluate genotype differences in locomotor activity rhythms, male Vip ${ }^{+/+}$, Vip $^{\text {cre/+}}$, and Vip ${ }^{\text {cre/cre }}$ mice (12-18 weeks old, $n=6-11 /$ genotype) were individually housed in running-wheel cages under LD12:12 
(lights-off at 1800 CST, white light-emitting diode lights, $310 \pm 80$ lux) for 24 days before release into constant darkness (DD) for 29 days. After DD, mice were reentrained to LD12:12 for 32 days and then exposed to simulated jetlag with a 6-h advance of the LD cycle (new lights-off: 1200 CST). After the shift of the LD cycle, mice were left undisturbed for 36 days, with the exception of routine husbandry. Wheel-running data were collected and analyzed with ClockLab software (Actimetrics, Wilmette, IL, USA). Activity onsets were determined daily, and free-running period was quantified using a linear regression fit to the first 10 days of DD. Precision was quantified as the inverse of the standard deviation of activity onset times over this same interval. Jetlag reentrainment was quantified for each mouse as the number of days required for the timing of activity onset to shift by $6 \mathrm{~h}$.

\section{Tissue Collection and Ex Vivo Assays}

To test Cre-induced changes in SCN function and peripheral tissues, male and female $\mathrm{Vip}^{+/+}$, Vip ${ }^{\text {cre/+ }}$, and $\mathrm{Vip}$ cre/cre mice (14-23 weeks old) were sacrificed 4 to $6 \mathrm{~h}$ before lights-off, as in Davidson et al., (2009). SCN slices $(150 \mu \mathrm{m})$ were sectioned in the coronal plane using a Leica VT1200S vibratome, whereas other tissues were trimmed by hand with a scalpel. Tissue samples were cultured at $37^{\circ} \mathrm{C}$ on a membrane insert in $1.2 \mathrm{~mL}$ of air-buffered Dulbecco's Modified Eagle's Medium (DMEM; SCN DMEM: Sigma D2902, non-SCN DMEM: Gibco 12100-046) supplemented with $0.1 \mathrm{mM}$ beetle luciferin, $0.02 \%$ B27 (Gibco 17504), 0.01\% HEPES (Gibco 15630), 0.005\% NaCHO 3 (Gibco 25080), 0.004\% dextrose (Sigma G7021), and 0.01\% penicillin/streptomycin (Gibco 15140). Bioluminescence rhythms were recorded for 5 to 6 days with a luminometer (Actimetrics, LumiCycle 32) or a Stanford Photonics XR Mega 10z charge-coupled device camera mounted onto a Zeiss Axio Observer Z1 microscope. For drug treatments, the culture medium was treated at the time of collection and left for the duration of the recording experiment. To test VIP signaling, SCN slices were cultured with vehicle or VPAC2 antagonist (4Cl-D-Phe6,Leu17), VIP (20 $\mathrm{MM}$, Tocris, cat No. 3054, $n=12-31 / g e n o t y p e / d r u g ~ c o n d i t i o n)$, as in Evans et al. (2013). To examine the contribution of AVP signaling, SCN slices were cultured with vehicle or a cocktail of AVP receptor antagonists (V1a receptor antagonist OPC21268 [100 uM, Tocris, cat No. 3924) + V1b receptor antagonist SSR149415 [100 uM, Axon Medchem cat No. 1116], $n=9-18 /$ genotype/drug condition), as in Bedont et al. (2018). Last, to test the efficacy of Cre-induced transduction, SCN slices were collected from 10-day-old Vip ${ }^{\text {cre/+ }}$ and Vip ${ }^{\text {cre/cre mice }(~} n=4-5 /$ genotype) and cultured with AAV8FLEX-tdTomato (University of North Carolina Vector Core). tdTomato expression was imaged daily to assess transduction efficiency in vitro for 9 days on a Nikon confocal microscope A1 (Nikon Instruments, Melville, NY, USA).

\section{PER2::LUC Analyses}

Lumicycle- and Matlab-based computational analyses were used to analyze SCN function at the network and cellular level, as in previous work (Evans et al., 2011, 2013). Recording start time was normalized for each sample to ZT. For whole-tissue analyses, the PER2::LUC time series was detrended and analyzed with Lumicycle software by fitting a damped sine wave to the first 4 full cycles in vitro. Goodness of fit, period, and damping rate (i.e., number of days for rhythm amplitude to decrease about 37\%) was recorded from the sine fit. In addition, we recorded daily times and values of peak and trough PER2::LUC to calculate cycle-to-cycle amplitude (peak - trough), average period, and precision (i.e., 1/standard deviation of cycle-to-cycle period). For spatiotemporal analyses, Matlab-based computational analyses were used (Evans et al., 2011, 2013). Briefly, a time series was generated for 
each 12-pixel-diameter ROI using a uniform grid with 2-pixel spacing, for which the linear trend was eliminated, and a Butterworth filter was applied to remove high- and low-frequency interference. To generate average maps for each group, samples were aligned to the same $X-Y$ coordinates by minimizing the sum of squared difference of the first 24-h summed bioluminescence profiles. Average maps were used to identify subregions for cellular analyses. To locate and extract data from cell-like ROIs, an iterative process was employed after background and local noise subtraction. For each celllike ROI, we extracted the average period, phase of peak PER2::LUC on the first cycle in vitro, and precision of cellular period. We also calculated the circular standard deviation of peak times for celllike ROIs in each SCN slice using Oriana software.

\section{Statistical Analyses}

Data are represented in figures as mean \pm SEM. Statistical analyses were performed with JMP software (SAS Institute, Cary, NC, USA). For most analyses, 1-way analysis of variance (ANOVA) was used to assess the effects of genotype, followed by post hoc Tukey's honestly significant difference (HSD) to control for familywise error. Changes in behavior and SCN function over time were analyzed with repeated-measures ANOVA, followed by post hoc least square means contrasts. Statistical significance was set at $p<0.05$ in all cases.

\section{Results}

\section{VIP-IRES-Cre Transgene Induces a Progressive Postnatal Loss of VIP Expression}

To test whether VIP expression is affected by the VIP-IRES-Cre transgene, we performed immunohistochemistry in brain slices collected from 10-day-old mice at ZT12 (Fig. 1A), an age when VIP expression is expected to be elevated (Carmona-Alcocer et al., 2018). At postnatal day 10 (P10), VIP expression in the SCN differed by genotype (1-way ANOVA, $F_{2,19}=3.57, p<0.05$ ) with about $50 \%$ reduction in Vip ${ }^{\text {cre/cre }}$ mice relative to Vip $^{\text {cre/+ }}$ and Vip ${ }^{+/+}$mice (Fig. 1B; Tukey's HSD, $p<0.05$ ). Despite reduced VIP, Cre was sufficient at this age to transduce both Vip ${ }^{\text {cre/+ }}$ and Vip ${ }^{\text {cre/cre }}$ SCN neurons (Suppl. Fig. S1; repeated-measures ANOVA, genotype: $F_{1,7}=0.06, p>0.8$; genotype $\times$ time: $F_{5,3}=$ $0.76, p>0.6)$. To test whether the deficit induced by VIP-IRES-Cre worsened with age, we next examined VIP expression in the SCN of adult mice administered an intracerebroventricular injection of colchicine to interrupt microtubule transport and visualize total protein across the circadian cycle. As seen at P10, VIP in the adult SCN was reduced by the insertion of the VIP-IRES-Cre transgene (1way ANOVA, $\left.F_{2,9}=27.33, p<0.0005\right)$, but at this age, it was decreased to a larger degree in VIP-IRES-Cre mutant mice and in rough proportion to copy number. Compared with $\mathrm{Vip}^{+/+} \mathrm{SCN}, \mathrm{VIP}$ was reduced by

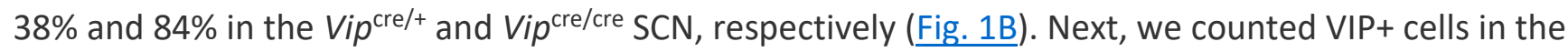
adult SCN to investigate if this reflected loss of VIP protein or VIP+ cells (Fig. 1C). The number of VIP+ cells differed by genotype (Fig. 1C; 1-way ANOVA, $F_{2,9}=24.4, p<0.0005$ ), being reduced by $85 \%$ in the homozygous $\mathrm{Vip}^{\text {cre/cre }} \mathrm{SCN}$ relative to $\mathrm{Vip}^{\text {cre/+ }}$ and Vip ${ }^{+/+} \mathrm{SCN}$ (Fig. 1C; Tukey's HSD, $p<0.001$ ). Although the number of VIP+ cells was reduced in Vip ${ }^{\text {cre/cre }}$ mice, DAPI staining in the ventral SCN did not differ by genotype (Fig. 1D; 1-way ANOVA, $F_{2,9}=0.93, p>0.3$ ), suggesting cells in this region were not lost due to Cre-induced neurotoxicity (Rezai Amin et al., 2019), which was consistent with effective Cre

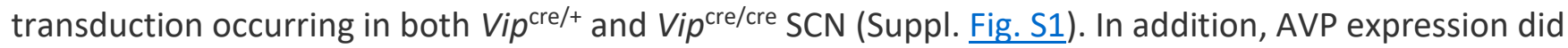
not differ between genotypes in P10 or adult mice (Suppl. Fig. S2; P10: 1-way ANOVA, $F_{2,19}=0.36, p>$ 0.7 ; adult: 1 way ANOVA, $\left.F_{2,9}=0.60, p>0.5\right)$. Taken together, these results indicate that the VIP-IRES- 
Cre transgene induces a progressive postnatal loss of VIP expression that affects both Vip ${ }^{\text {cre/+}}$ and Vip ${ }^{\text {cre/cre }}$ mice in adulthood.
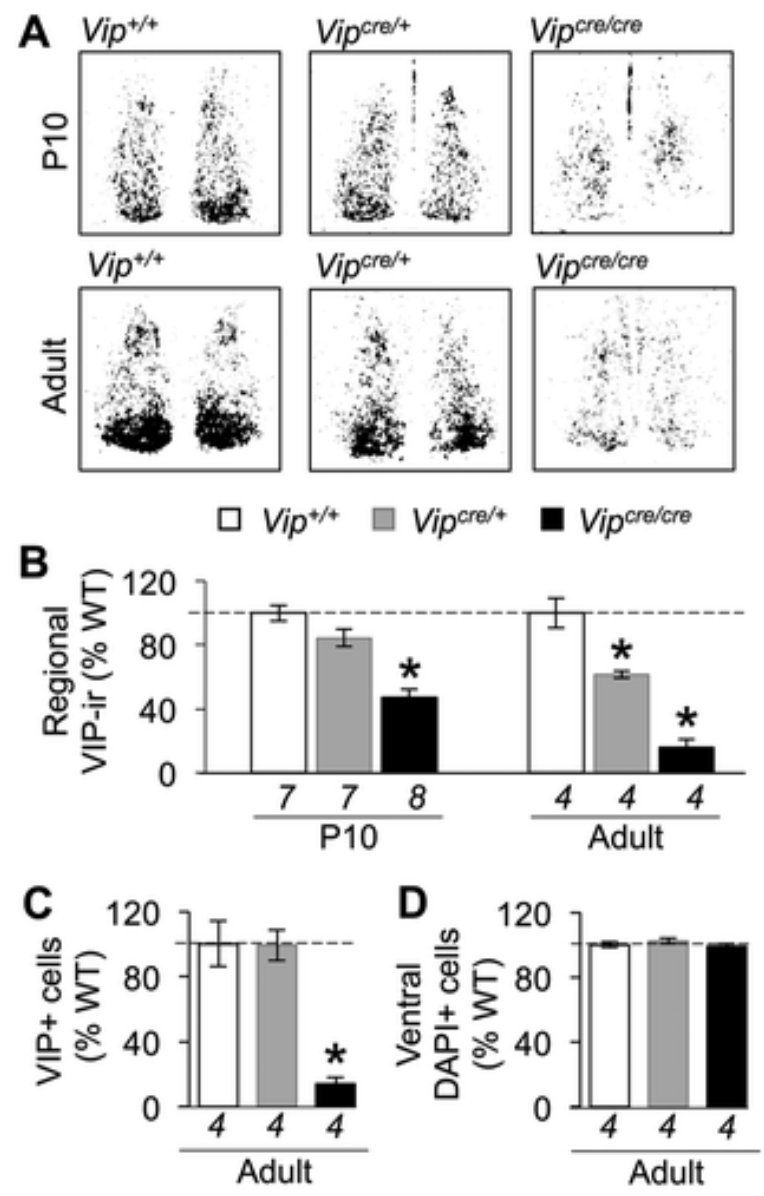

Figure 1. The VIP-IRES-Cre transgene decreases vasoactive intestinal polypeptide (VIP) expression in proportion to copy number in an age-related manner. (A) Representative, thresholded images of VIP expression in the SCN of $\mathrm{Vip}^{+/+}, \mathrm{Vip}^{\text {cre/+}}$, and $\mathrm{Vip}^{\text {cre/cre }}$ mice collected at P10 or in adulthood. (B) The VIP-IRES-Cre transgene causes an age-related decrease in regional VIP levels in the SCN. (C) The VIP-IRES-Cre transgene reduces the number of VIP+ cells in the SCN of adult Vip ${ }^{\text {cre/cre }}$ mice but not in adult Vip ${ }^{\text {cre/+ }}$ mice. (D) The VIP-IRES-Cre transgene does not reduce DAPI-labeled cells in the ventral SCN. Italicized numbers below the abscissa indicate sample size. *Differs from Vip ${ }^{+/+}$, Tukey's HSD, $p<0.05$.

Vip ${ }^{\text {cre/cre }}$ Mice Exhibit a Circadian Phenotype Consistent with Other VIP Deficient Models We next assessed the behavioral phenotype of Vip ${ }^{\text {cre/cre }}$ mice to compare it with other models deficient in VIP signaling (Vosko et al., 2007). First, we evaluated changes in intrinsic timekeeping by examining locomotor rhythms of adult male $\mathrm{Vip}^{\mathrm{cre} / \mathrm{cre}}, \mathrm{Vip}^{\mathrm{cre} /+}$, and $\mathrm{Vip}^{+/+}$mice in constant darkness (DD; Fig. 2A). All mice retained coherent wheel-running rhythms during the 4 weeks of $D D$, but genotype differences were detected in both the precision (1-way ANOVA, $F_{2,20}=13.38, p<0.0005$ ) and period of freerunning rhythms (1-way ANOVA, $\left.F_{2,20}=11.97, p<0.0005\right)$. Relative

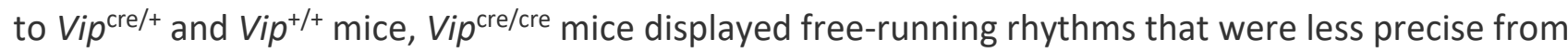
day to day (Fig. 2B; Tukey's HSD, $p<0.007$ ) with a shorter period (Fig. 2C; Tukey's HSD, $p<0.02$ ). Second, we tested whether VIP-Cre mice would respond differently to simulated jetlag (Fig. 2D) since loss of VIP signaling has been reported to alter circadian responses to light (Vosko et al., 2007). After an abrupt 6-h advance of the light:dark cycle, reentrainment to the new photocycle differed by 
genotype (1-way ANOVA, $\left.F_{2,20}=3.68, p<0.05\right)$. Vip ${ }^{\text {cre/cre }}$ mice required fewer days to reentrain

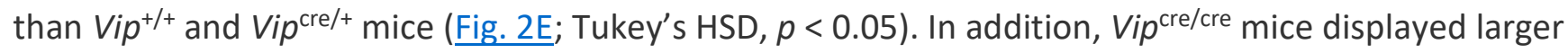
phase shifts on each of the first 4 days of simulated jetlag (Fig. 2F-G; repeated-measures ANOVA, $F_{2,20}=$ 8.24, $p<0.005$; LSM contrasts, $p<0.05$ ), with a nonlinear pattern suggesting that accelerated recovery was not merely due to their shorter period. Taken together, these data indicate that the loss of VIP in homozygous VIP-IRES-Cre mutant mice alters the intrinsic function of the circadian clock and its response to photic stimuli, which are phenotypes likewise observed in other VIP-deficient mouse models.
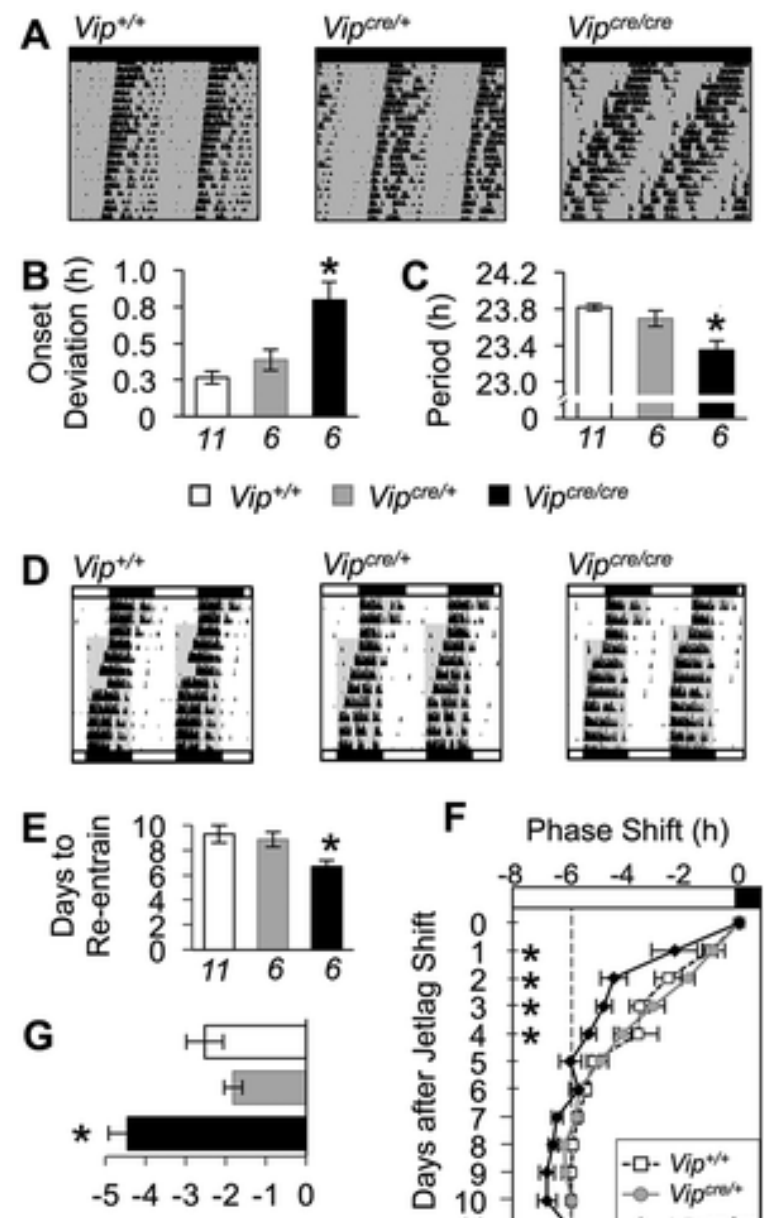

Day 2 Phase Shift (h)

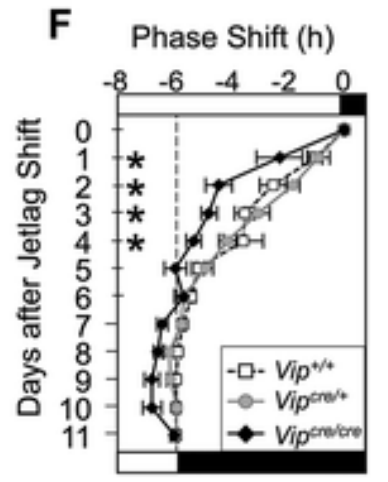

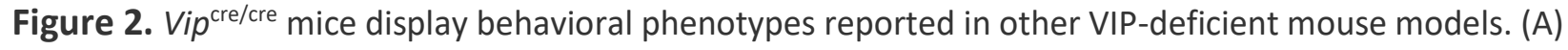
Representative double-plotted wheel-running actograms illustrating locomotor rhythms in constant darkness. Lighting conditions illustrated by the bar above each actogram (black = darkness, white $=$ light) and by internal shading. (B,. C) Vip cre/cre mice have less precise rhythms (B) and free-running rhythms with shorter period (C). (D) Representative double-plotted wheel-running actograms illustrating response to simulated jetlag. (E-

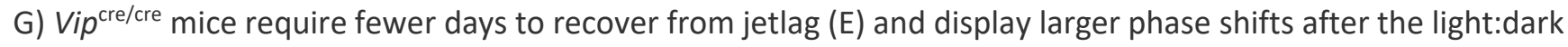
cycle shift (F-G). Vertical dashed line in panel (F) indicates the phase shift required for reentrainment to the 6-h advance of the light:dark cycle. Italicized numbers below the abscissa in (B) indicate the sample size for all related panels. *Differs from Vip ${ }^{+/}$, Tukey's HSD, $p<0.05$. 
The Vip ${ }^{\text {cre/cre }}$ SCN Displays a Shorter Period, but PER2::LUC Rhythms Are Otherwise Intact Because the behavioral phenotype caused by loss of VIP signaling in $\mathrm{Vip}^{-/}$and $\mathrm{Vipr}^{-1-}$ mouse models is associated with changes in SCN function (Aton et al., 2005; Ciarleglio et al., 2009), we next determined if Cre-induced loss of VIP alters master clock function. All SCN collected displayed self-sustained PER2::LUC rhythms (Fig. 3A), but SCN period varied by genotype (1-way ANOVA, $F_{2,85}=7.3, p<0.005$ ). Similar to differences in vivo, period was shorter in Vip cre/cre SCN than in Vip ${ }^{+/+}$SCN (Fig. 3B; Tukey's HSD, $p<0.005$ ). However, Vip $p^{\text {cre/cre }} \mathrm{SCN}$ did not differ from $\mathrm{Vip}^{+/+} \mathrm{SCN}$ in either the precision (Fig. 3C; 1 way ANOVA, $F_{2,84}=1.89, p>0.1$ ) or the amplitude of SCN rhythms ( $\underline{\text { Fig. } 3 \mathrm{D}}$; repeated-measures ANOVA, genotype: $F_{2,84}=0.71, p>0.4$; time $\times$ genotype: $F_{2,164}=1.57, p>0.1$ ). The period of peripheral clock tissues did not differ by genotype (Suppl. Fig. S3), suggesting that effects of VIP-IRES-Cre were specific to the SCN clock (adrenal: 1 -way ANOVA, $F_{2,16}=2.87, p=0.09$; liver: 1-way ANOVA, $F_{2,17}=$ $0.66, p>0.5$; lung: 1-way ANOVA, $\left.F_{2,18}=0.66, p>0.5\right)$.
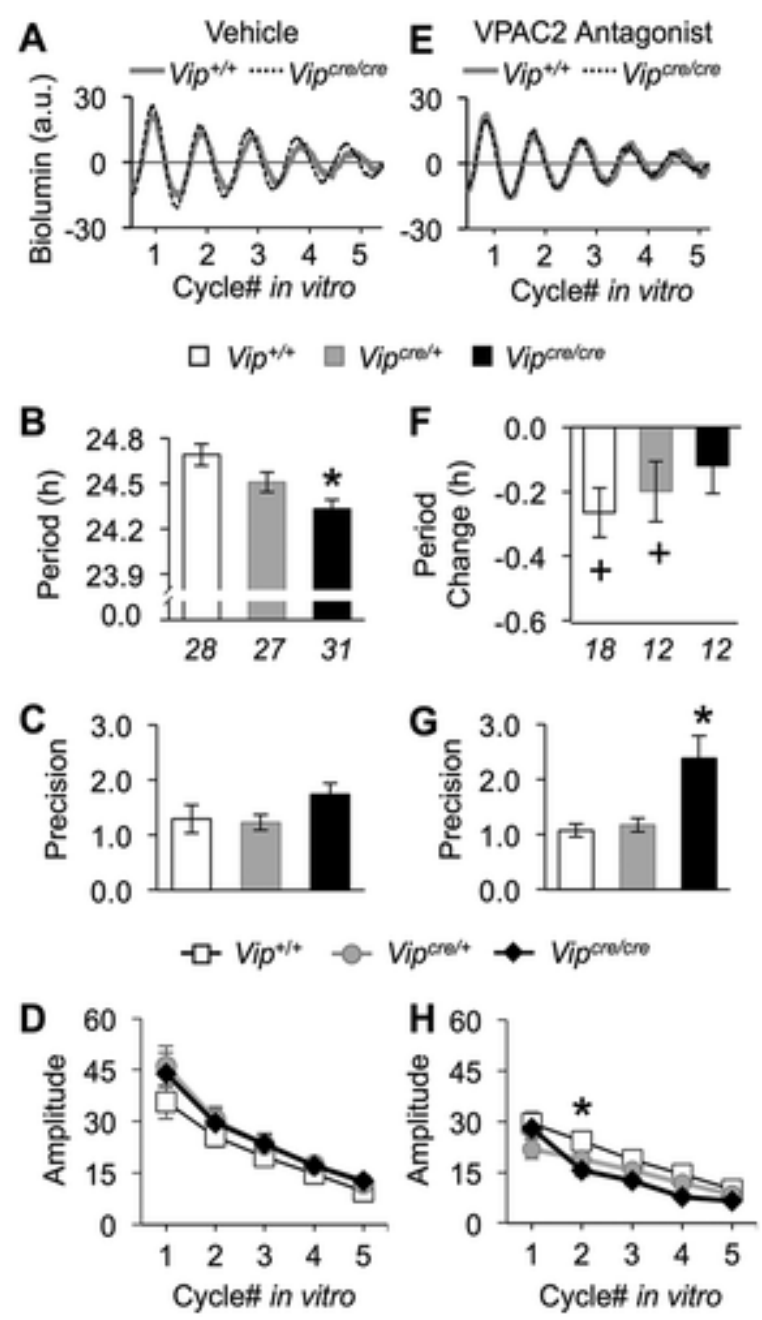

Figure 3. Molecular rhythms in the SCN of Vip cre/cre mice are altered due to the loss of VIP signaling. (A) Representative PER2::LUC time series illustrating SCN bioluminescence rhythms under standard culture conditions. For the color version, see Supplementary Figure S4. (B-D) The SCN of Vip cre/cre mice display PER2::LUC rhythms with a shorter period (B), but precision (C) and amplitude (D) did not differ by genotype. (E) Representative PER2::LUC time series illustrating SCN bioluminescence rhythms during VPAC2 antagonism. (F) VPAC2 inhibition did not shorten period in Vip ${ }^{\text {cre/cre }} S C N .(G, H)$ Precision increased in Vip ${ }^{\text {cre/cre }}$ SCN during VPAC2 inhibition (G), but amplitude was not markedly affected $(H)$. Italicized numbers below the abscissa in (B) and (F) 
indicate the sample size for all related panels. * Differs from Vip ${ }^{+/+}$(same condition): Tukey's HSD, $p<0.05 ;+$ differs from vehicle control (within genotype): one-sample $t$ test, $p<0.05$.

Residual VIP Signaling Does Not Drive Intact PER2::LUC Rhythms in Vip ${ }^{\text {cre/cre }}$ SCN

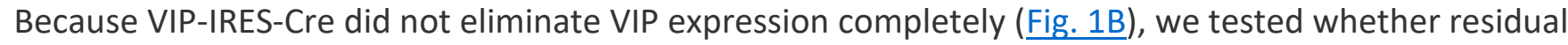
VIP signaling sustained SCN rhythms by culturing slices with the VPAC2 antagonist, (4Cl-D-Phe6,Leu17) VIP (20 $\mathrm{MM}$, as in Evans et al., 2013). Similar to vehicle conditions, all SCN slices displayed PER2::LUC rhythms for at least 5 days in culture (Fig. 3E). As expected, VPAC2 antagonism shortened Vip $^{+/+} \mathrm{SCN}$ period (Fig. 3F; 1-sample $t$ test, $t[17]=3.44, p<0.005$ ) and tended to shorten period in the Vip ${ }^{\text {cre } /+} \mathrm{SCN}$ (Fig. $3 \mathrm{~F}, 1$-sample $t$ test, $t[11]=2.11, p=0.058$ ). In contrast, period was not affected in Vip ${ }^{\text {cre } / c r e ~} S C N$ (Fig. 3F; 1-sample $t$ test, $t[11]=1.37, p>0.15$ ), and genotypic differences in the response to VPAC2 inhibition equalized SCN period across groups (1-way ANOVA, $F_{2,39}=1.66, p>0.2$ ). VPAC2 antagonism modulated SCN precision (1-way ANOVA, $F_{2,39}=9.63, p<0.0005$ ), but this treatment increased

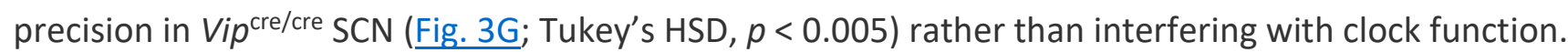
Last, VPAC2 antagonism modulated the amplitude of PER2::LUC rhythms by genotype (repeatedmeasures ANOVA, genotype: $F_{2,39}=2.0, p>0.1$; time $\times$ genotype: $F_{8,74}=5.61, p<0.0001$ ) by decreasing the amplitude in homozygous VIP-Cre specifically on cycle 2 in vitro and not other cycles (Fig. 3H; LSM contrasts, $p<0.01$ ). In sum, these data suggest that residual VIP signaling is not required for PER2::LUC rhythms in Vip cre/cre SCN.

\section{Region-Specific Changes in Cellular Period and Phase in the Vip ${ }^{\text {cre/cre }}$ SCN}

To further explore how VIP-IRES-Cre may affect SCN function, slices were collected for real-time bioluminescence imaging of cellular PER2::LUC rhythms (Fig. 4A). The total number of rhythmic cells in SCN slices differed by genotype (1-way ANOVA, $F_{2,15}=4.53, p<0.05$ ), with $20 \%$ fewer rhythmic cells in Vip ${ }^{\text {cre/cre }}$ slices (Fig. 4B; Tukey's HSD, $p<0.05$ ) and a reduced number of rhythmic cells in both the dorsal and ventral SCN (Fig. 4C; dorsal: 1-way ANOVA, $F_{2,15}=5.38$, ventral: 1 -way ANOVA, $F_{2,15}=8.20$, Tukey's HSD, $p<0.05$ in each case). As expected from luminometry analyses (Fig. 3B), SCN period in imaged samples differed by genotype (Fig. 4D; full factorial ANOVA, genotype: $F_{2,798}=5.44, p<0.005$ ), with a trend for an interaction by SCN region (genotype $\times$ region: $F_{2,798}=2.38, p=0.09$; region: $F_{1,798}=$ $1.71, p>0.1)$. Specifically, ventral SCN cells displayed a shorter period in Vip ${ }^{\text {cre/cre }}$ slices relative to $\mathrm{Vip}^{+/+}$slices (Fig. 4D; LSM contrasts, $p=0.0005$ ), but cellular period in the dorsal SCN did not differ by genotype (Fig. 4D; LSM contrasts, $p>0.1$ ). In addition, cellular relationships in the SCN differed by genotype (Fig. 4E, full factorial ANOVA, genotype: $F_{2,798}=0.93, p=0.3958$; region: $F_{1,798}=14.1261, p=$ 0.0002; genotype $\times$ region: $\left.F_{2,798}=12.4203, p<0.0001\right)$, with regional phase differences attenuated in both $\mathrm{Vip}^{\text {cre/cre }}$ and $\mathrm{Vip}^{\text {cre/+ }} \mathrm{SCN}$ due to changes in the phase of both dorsal and ventral cells (Fig. 4E; LSM contrasts, $p<0.05$ ). Taken together, these data suggest that the presence of the VIP-IRES-Cre transgene alters SCN network organization in VIP-Cre mice, although PER2::LUC rhythms remain intact. 

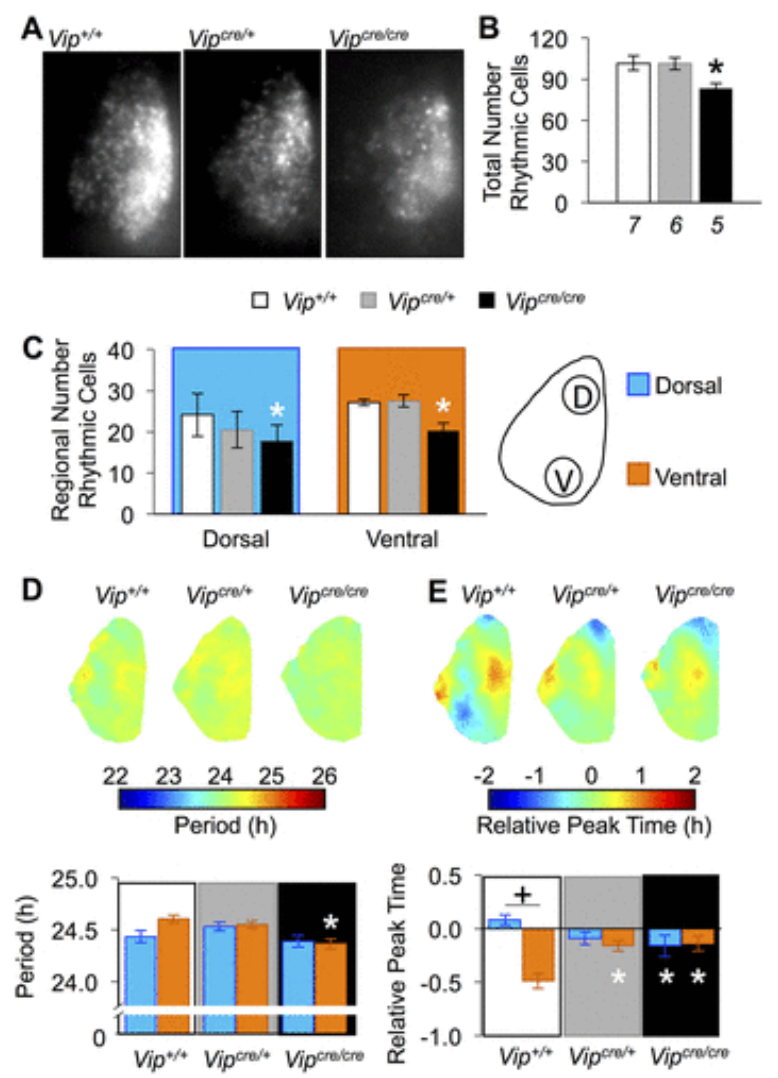

Figure 4. SCN network function is altered in Vip ${ }^{\text {cre/cre }}$ mice. (A) Representative bioluminescence images illustrating summed PER2::LUC expression over the first $24 \mathrm{~h}$ of recording. (B) The total number of rhythmic cells was reduced in Vip ${ }^{\text {cre/cre }}$ SCN. (C) Rhythmic cells were reduced in both the dorsal and ventral SCN of $\mathrm{Vip}^{\text {cre/cre }}$ mice. Schematic illustrates dorsal and ventral regions used for quantification. (D) Period maps and cellular period data demonstrate shorter period in the ventral SCN in Vip ${ }^{\text {cre/cre }}$ mice. (E) Phase maps and cellular phase data demonstrate regional phase differences are attenuated in Vip ${ }^{\text {cre/cre }} \mathrm{SCN}$. Italicized numbers below the abscissa in (B) indicate the number of SCN slices for each genotype (cellular sample size $=89-184 \mathrm{SCN}$ cells/region). *Differs from the same region in $\mathrm{Vip}^{+/+}$, LSM contrasts, $p<0.05$; + differs from complementary SCN region (within genotype), LSM contrasts, $p<0.05$.

\section{AVP Signaling Contributes to Both Intracellular and Intercellular Clock Function in the $\mathrm{Vip}^{\text {cre/cre }} \mathrm{SCN}$}

Given that SCN function is largely intact in Vip ${ }^{\text {cre/cre }}$ mice, we next tested the hypothesis that other neuropeptides help to sustain clock function in this model. We examined the potential contribution of AVP because this neuropeptide is decreased in $\mathrm{Vip}^{-/-}$and $\mathrm{Vipr}^{-{ }^{--}}$knockout mice but not VIP-Cre mice (Suppl. Fig. S2). To test the role of AVP signaling, PER2::LUC slices were cultured with either vehicle or a cocktail of antagonists for the AVP receptors V1a and V1b (100 $\mu \mathrm{M}$, OPC21268 + SSR149415). AVP receptor antagonism lowered PER2::LUC amplitude in a genotype-dependent manner ( $\mathrm{Fig} .5 \mathrm{~A}, \mathrm{~B}$; repeated-measures ANOVA, genotype: $F_{2,38}=13.63, p<0.0001$; genotype $\times$ time: $\left.F_{8,48}\right)=2.14, p<$ $0.05)$, with reduced amplitude in both Vip ${ }^{\text {cre/cre }}$ and Vip ${ }^{\text {cre/+ }}$ SCN (Fig. 5A, B; LSM contrasts, $p<0.01$ ). V1 antagonists also affected SCN precision (Fig. 5C; 1 -way ANOVA $F_{2,32}=5.16, p<0.05$ ), with reductions in both Vip ${ }^{\text {cre/cre }}$ and Vip ${ }^{\text {cre/+ }}$ SCN (Fig. 5C; Tukey's HSD, $p<0.05$ ). We next conducted bioluminescence imaging to test whether $\mathrm{V} 1$ antagonist effects were due to intra- or intercellular deficits in clock function (Fig. 5D). V1 antagonists markedly suppressed the PER2::LUC signal in Vip ${ }^{\text {cre/cre }}$ SCN, with 
deficits evident at both the cellular and population level (Fig. 5E). Specifically, AVP receptor antagonism

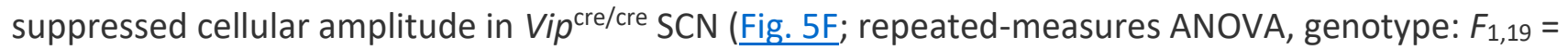
12.6, $p<0.005$ genotype $\times$ time: $F_{4,16}=4.8, p<0.01$ ) and increased the standard deviation of cellular peak times (Fig. 5G; repeated-measures ANOVA, genotype: $F_{1,19}=8.02, p<0.05$; genotype $\times$ time: $\left.F_{4,16}=3.8, p<0.05\right)$. Importantly, neither cellular amplitude nor synchrony differed by genotype under vehicle conditions (repeated-measures ANOVA, $p>0.1$ in each case). Collectively, these results suggest that AVP signaling contributes to cellular clock function and network synchrony in the SCN of VIP-IRES-Cre mutants.

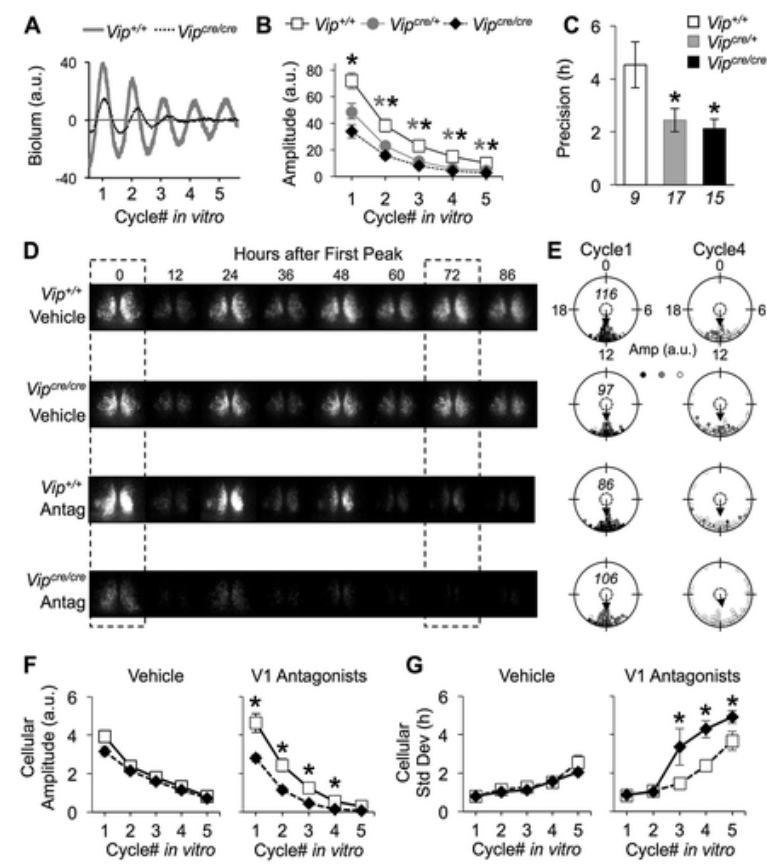

Figure 5. Arginine vasopressin (AVP) signaling maintains the amplitude and synchrony of Vip ${ }^{\text {cre/cre }}$ SCN rhythms. (A) Representative PER2::LUC time series illustrating SCN bioluminescence rhythms during AVP receptor antagonism. For the color version, see Supplementary Figure S4. (B, C) AVP receptor antagonism decreased the amplitude (B) and precision (C) of PER2::LUC rhythms in the Vip ${ }^{\text {cre/cre }}$ and Vip ${ }^{\text {cre/+ }}$ SCN. (D) Representative PER2::LUC bioluminescence images under vehicle (top) and AVP receptor antagonist (bottom) conditions. The dashed boxes indicate the start of cycle 1 and cycle 4 of the recording. (E) Raleigh plots depicting cellular peak times on the first cycle (cycle 1) and fourth cycle (cycle 4) in vitro for each sample in (D). The amplitude of the PER2::LUC rhythms for each cell is represented by color saturation (darker = larger amplitude). The italicized number within a plot indicates the number of cells for each sample. (F) AVP receptor antagonism decreased the amplitude of cellular PER2::LUC rhythms in Vip ${ }^{\text {cre/cre }}$ SCN. (G) AVP receptor antagonism increased the standard deviation of cellular peak times in Vip ${ }^{\text {cre/cre }}$ SCN slices. Italicized numbers below the abscissa in (C) indicate the number of SCN slices in (B) and (C). For (F-G), $n=3$ to $4 \mathrm{SCN}$ slices/genotype/condition (cellular sample size = 86$124 \mathrm{SCN}$ cells/slice). ${ }^{*}$ Differs from $\mathrm{Vip}^{+/+}$, LSM contrasts, $p<0.05$.

\section{Discussion}

Use of the Cre/loxP system has accelerated discovery in neuroscience, but it has been recognized for some time that transgene insertion may impose a genetic lesion (Taniguchi et al., 2011). Of note, the VIP-IRES-Cre model was created using methods designed to minimize interference with native protein expression (i.e., knock-in allele inserted after 3' stop codon including IRES sequence). Nevertheless, we show here that VIP is decreased in the SCN of both homozygous and heterozygous VIP-Cre mice, the 
latter of which is in agreement with a recent study (Cheng et al., 2019). We build on this previous work by including homozygous VIP-IRES-Cre mice in our analyses, employing PER2::LUC assays of clock function, and testing the role of AVP signaling in this VIP-deficient model. Importantly, we find that adult homozygous VIP-Cre mice display behavioral properties similar to those reported in VIP knockout mice in some respects (i.e., shorter period, faster reentrainment, and decreased precision) but not others (i.e., DD arrhythmia or phase jump upon DD release). Consistent with their behavioral phenotype, homozygous VIP-Cre mice display intact SCN PER2::LUC rhythms, albeit with altered period and network organization. We provide evidence that residual VIP signaling does not drive intact PER2::LUC rhythms in the homozygous VIP-Cre SCN, but rather this reflects a contribution of other signals such as AVP. Overall, these data provide a cautionary tale for investigators using this mouse model and new potential insight into the relative contribution of VIP and other neuropeptides in sustaining SCN function. The implications of these findings should be considered when designing, interpreting, and reporting work using the VIP-IRES-Cre model.

The present results indicate that VIP-Cre mice display a progressive postnatal loss of VIP, with decreased expression detected in homozygous VIP-Cre mice as early as P10. This reduction becomes more severe in adulthood, when it is also observed in heterozygous VIP-Cre mice. Although P10 mice were examined at a single time point, adult mice were given colchicine injections to evaluate total protein expression across the circadian day, as in other work (Varadarajan et al., 2018). Thus, it is possible that both VIP amount and timing are disturbed in adult heterozygous and homozygous VIP-Cre mice. Although the exact mechanism of VIP-Cre interference remains to be determined, it has been shown that Vip mRNA is reduced in 5- to 8-week-old heterozygous VIP-Cre mice (Cheng et al., 2019). This suggests that interference occurs at the transcriptional level, although another possibility is Crerelated cellular toxicity (Rezai Amin et al., 2019). Although we did not assess cell death directly, the current results suggest that reduced VIP expression in VIP mutants reflects the loss of protein synthesis rather than VIP+ cells. For instance, VIP was reduced by $38 \%$ in heterozygous VIP-Cre mice, but the number of VIP+ cells was similar to that seen in wild-type mice. In contrast, the number of VIP+ cells was reduced in homozygous VIP-Cre mice; however, this may not reflect a loss of cells because it is difficult to count cells with very low VIP levels using immunohistochemistry. Further evidence inconsistent with the loss of VIP+ cells was that the number of DAPI+ cells in the ventral SCN did not differ by genotype and Cre recombinase was sufficient to induce transduction in both heterozygous and homozygous VIP-Cre SCN. These data suggest that the largest effect of the VIP-Cre transgene is to reduce VIP synthesis, although it remains possible that loss of VIP+ cells occurs in homozygous VIP-Cre SCN. Taken together, our work suggests that VIP deficiency in the VIP-Cre mouse is developmentally acquired, with the timing and severity of VIP loss dependent on transgene copy number.

The conclusion that the VIP-IRES-Cre transgene does interfere with VIP signaling is also supported by the observation that adult homozygous VIP-Cre mice exhibit circadian phenotypes that have been reported to occur in other VIP-deficient mouse models (Vosko et al., 2007). Specifically, homozygous VIP-Cre mice exhibit locomotor rhythms that are shorter, less precise, and less robust, but they do not become arrhythmic in constant darkness. Consistent with the behavioral phenotype, SCN PER2::LUC rhythms in homozygous VIP-Cre mice are shorter and less robust but are not lost in the first week ex vivo. Although VIP loss is often expected to cause arrhythmia and SCN desynchrony, it is notable that arrhythmia is not always observed in $\mathrm{Vip}^{-/-}$and $\mathrm{Vipr}^{-/-}$knockout mice. For instance, some studies have 
reported that only $25 \%$ of VIP knockout mice become arrhythmic under constant darkness (Colwell et al., 2003), and other studies have found fragmented rhythms in $60 \%$ to $65 \%$ of VIP-deficient mice (Aton et al., 2005). Interestingly, heterozygous VIP-Cre mice do not display a behavioral phenotype despite reduced VIP expression (present study; Cheng et al., 2019), suggesting that 1 VIP-Cre allele is not sufficient to alter circadian behavior. This could be seen as very encouraging because at least half the circadian studies published to date explicitly use heterozygous VIP-Cre mice (Enoki et al., 2017; Fan et al., 2015; Hermanstyne et al., 2016; Jones et al., 2018; Lee et al., 2015; Liu et al., 2018; Mazuski et al., 2018; Mei et al., 2018; Miller et al., 2014; Todd et al., 2018). However, many studies published in the field do not provide critical information about the Cre model used, such as transgene copy number and strain stock number. Because we did detect differences in clock function in heterozygous VIP-Cre SCN during imaging and V1 antagonism studies, these data potentially unmask more subtle effects of insertion of a single VIP-IRES-Cre transgene. Thus, explicit reporting of Cre copy number, strain stock number, and inclusion of Cre-only controls will help facilitate interpretation of results obtained with this model. Further, we have examined only 1 VIP-IRES-Cre mouse line in the present work, but our results are consistent with reports demonstrating that the transgene in other Cre models can reduce native protein expression and cause physiological/behavioral phenotypes (Hoffmann et al., 2019; Viollet et al., 2017). It remains to be determined whether this issue likewise affects other VIPIRES-Cre lines and if other VIP-dependent processes are affected.

Given the marked reduction of VIP observed in the SCN of homozygous VIP-Cre mice, it was surprising that behavioral and SCN rhythms were intact. Blocking VPAC2 signaling for a week in vitro did not disrupt clock function in homozygous VIP-Cre SCN, suggesting residual VIP was not needed to sustain SCN rhythms over the week in vitro. Together with previous work, this suggests that absolute levels of VIP are not the sole factor in determining behavioral and cellular response to its deficiency. This is of particular interest given previous research demonstrating that SCN function can be disrupted when there is too little VIP (Aton et al., 2005), too much VIP (An et al., 2013), or if it is not timed correctly (Ananthasubramaniam et al., 2014). That VIP loss in homozygous VIP-Cre mice was not more disruptive to circadian timekeeping at either the behavioral or SCN level may reflect the progressive acquisition of the deficiency during postnatal development, which differs from Vip ${ }^{-/}$and Vipr2 ${ }^{-/-}$knockout models. Work investigating VIP-specific deficiency in adulthood is limited (Brown et al., 2005), so future work may test the effects of VIP deficiency specifically in adult mice.

Many SCN signals other than VIP are affected in germline VIP-deficient mouse models (Bedont et al., 2018; Harmar et al., 2002; Prosser et al., 2007; Varadarajan et al., 2018), but to our knowledge, the potential contribution of these other signals to the phenotype of VIP-deficient mice has not been tested directly. We show here that blockade of AVP signaling significantly disrupts clock function in the VIP-Cre SCN, indicating that AVP signaling can contribute to SCN function in the absence of VIP. This result is interesting given that previous work suggests that VIP is not strictly necessary for circadian function (Colwell et al., 2003; Aton et al., 2005). In addition, a variety of interventions can restore rhythmicity in mice lacking VIP signaling (Hughes et al., 2015; Power et al., 2010), indicating that other signaling factors can compensate for VIP loss. That AVP directly influences the function of the SCN network is consistent with previous work (Bedont et al., 2018; Edwards et al., 2016; Maywood et al., 2011). Together with our current findings, this suggests that other SCN signals may be leveraged to offset adverse consequences of VIP deficiency. 
In summary, we find that the Cre transgene interferes with VIP expression in VIP-Cre mutant mice, which should be an important consideration when using this model to investigate cell-type-specific contributions. On the other hand, homozygous VIP-Cre mice retain daily rhythms in locomotor behavior and SCN PER2::LUC expression despite displaying very low VIP levels. It is well appreciated that VIP is an important neuropeptide that regulates circadian function, but the SCN contains many other cell types whose roles are not well understood (Wen et al., 2020). One interesting possibility is that distinct signals regulate different emergent properties of the SCN network (e.g., period synchrony, phase relationships, precision/amplitude modulation) that contribute to master clock function in

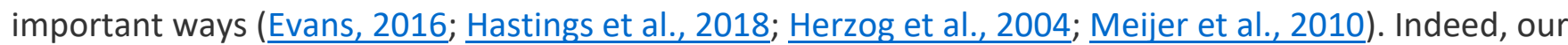
results establish that in the absence of VIP, other signals contribute to the maintenance of SCN clock function in vivo and in vitro. As such, the present results uncover a potential caveat working with VIPCre mice but also highlight new aspects of SCN circuits that are difficult to investigate using germline $\mathrm{Vip}^{-{ }^{-}}$and $\mathrm{Vipr}^{-\mathrm{I}^{-}}$knockouts.

\section{Acknowledgements}

We would like to thank Alec Huber, Austin Fritsch, Alecia Bjerke, Erika Johnson, Audrey Konieczny, and Berri Foreman for animal care. We are also grateful to Stanford Photonics for their equipment and technical assistance. The authors disclosed receipt of the following financial support for the research, authorship, and/or publication of this article: This work was supported by the National Institutes of Health (R01091234), the Whitehall Foundation (2014-12-65), and the Charles E. Kubly Mental Health Research Center.

\section{Conflict of Interest Statement}

The authors have no potential conflicts of interest with respect to the research, authorship, and/or publication of this article.

\section{Note}

Supplementary material is available for this article online.

\section{References}

An, S, Harang, R, Meeker, K, Granados-Fuentes, D, Tsai, CA, Mazuski, C, Kim, J, Doyle, FJ, Petzold, LR, Herzog, ED (2013) A neuropeptide speeds circadian entrainment by reducing intercellular synchrony. Proc Natl Acad Sci USA 110:E4355-4361.

Ananthasubramaniam, B, Herzog, ED, Herzel, H (2014) Timing of neuropeptide coupling determines synchrony and entrainment in the mammalian circadian clock. PLoS Comput Biol 10:e1003565.

Aton, SJ, Colwell, CS, Harmar, AJ, Waschek, J, Herzog, ED (2005) Vasoactive intestinal polypeptide mediates circadian rhythmicity and synchrony in mammalian clock neurons. Nat Neurosci 8:476-483.

Bedont, JL, Rohr, KE, Bathini, A, Hattar, S, Blackshaw, S, Sehgal, A, Evans, JA (2018) Asymmetric vasopressin signaling spatially organizes the master circadian clock. J Comp Neurol 526:20482067. 
Brown, TM, Hughes, AT, Piggins, HD (2005) Gastrin-releasing peptide promotes suprachiasmatic nuclei cellular rhythmicity in the absence of vasoactive intestinal polypeptide-VPAC2 receptor signaling. J Neurosci 25:11155-11164.

Buhr, ED, Takahashi, JS (2013) Molecular components of the mammalian circadian clock. Handb Exp Pharmacol 217:3-27.

Carmona-Alcocer, V, Rohr, KE, Joye, DAM, Evans, JA (2018) Circuit development in the master clock network of mammals. Eur J Neurosci 51(1):82-108.

Cheng, AH, Fung, SW, Cheng, HM (2019) Limitations of the Avp-IRES2-Cre (JAX \#023530) and VipIRES-Cre (JAX \#010908) models for chronobiological investigations. J Biol Rhythms 34(6):634644.

Ciarleglio, CM, Gamble, KL, Axley, JC, Strauss, BR, Cohen, JY, Colwell, CS, McMahon, DG (2009) Population encoding by circadian clock neurons organizes circadian behavior. $J$ Neurosci 29:1670-1676.

Colwell, CS, Michel, S, Itri, J, Rodriguez, W, Tam, J, Lelievre, V, Hu, Z, Liu, X, Waschek, JA (2003) Disrupted circadian rhythms in VIP- and PHI-deficient mice. Am J Physiol Regul Integr Comp Physiol 285:R939-R949.

Cutler, DJ, Haraura, M, Reed, HE, Shen, S, Sheward, WJ, Morrison, CF, Marston, HM, Harmar, AJ, Piggins, HD (2003) The mouse VPAC2 receptor confers suprachiasmatic nuclei cellular rhythmicity and responsiveness to vasoactive intestinal polypeptide in vitro. Eur J Neurosci 17:197-204.

Dardente, H, Menet, JS, Challet, E, Tournier, BB, Pevet, P, Masson-Pevet, M (2004) Daily and circadian expression of neuropeptides in the suprachiasmatic nuclei of nocturnal and diurnal rodents. Brain Res Mol Brain Res 124:143-151.

Davidson, AJ, Castanon-Cervantes, O, Leise, TL, Molyneux, PC, Harrington, ME (2009) Visualizing jet lag in the mouse suprachiasmatic nucleus and peripheral circadian timing system. Eur J Neurosci 29:171-180.

Edwards, MD, Brancaccio, M, Chesham, JE, Maywood, ES, Hastings, MH (2016) Rhythmic expression of cryptochrome induces the circadian clock of arrhythmic suprachiasmatic nuclei through arginine vasopressin signaling. Proc Natl Acad Sci USA 113:2732-2737.

Enoki, R, Oda, Y, Mieda, M, Ono, D, Honma, S, Honma, KI (2017) Synchronous circadian voltage rhythms with asynchronous calcium rhythms in the suprachiasmatic nucleus. Proc Natl Acad Sci USA 114:E2476-E2485.

Evans, JA (2016) Collective timekeeping among cells of the master circadian clock. J Endocrinol 230:R27-R49.

Evans, JA, Leise, TL, Castanon-Cervantes, O, Davidson, AJ (2011) Intrinsic regulation of spatiotemporal organization within the suprachiasmatic nucleus. PLoS One 6:e15869.

Evans, JA, Leise, TL, Castanon-Cervantes, O, Davidson, AJ (2013) Dynamic interactions mediated by nonredundant signaling mechanisms couple circadian clock neurons. Neuron 80:973-983.

Fan, J, Zeng, H, Olson, DP, Huber, KM, Gibson, JR, Takahashi, JS (2015) Vasoactive intestinal polypeptide (VIP)-expressing neurons in the suprachiasmatic nucleus provide sparse GABAergic outputs to local neurons with circadian regulation occurring distal to the opening of postsynaptic GABAA ionotropic receptors. J Neurosci 35:1905-1920.

Harmar, AJ, Marston, HM, Shen, S, Spratt, C, West, KM, Sheward, WJ, Morrison, CF, Dorin, JR, Piggins, HD, Reubi, JC, et al. (2002) The VPAC(2) receptor is essential for circadian function in the mouse suprachiasmatic nuclei. Cell 109:497-508. 
Hastings, MH, Maywood, ES, Brancaccio, M (2018) Generation of circadian rhythms in the suprachiasmatic nucleus. Nat Rev Neurosci 19:453-469.

Hermanstyne, TO, Simms, CL, Carrasquillo, Y, Herzog, ED, Nerbonne, JM (2016) Distinct firing properties of vasoactive intestinal peptide-expressing neurons in the suprachiasmatic nucleus. J Biol Rhythm 31:57-67.

Herzog, ED, Aton, SJ, Numano, R, Sakaki, Y, Tei, H (2004) Temporal precision in the mammalian circadian system: a reliable clock from less reliable neurons. J Biol Rhythms 19:35-46.

Hoffmann, HM, Larder, R, Lee, JS, Hu, RJ, Trang, C, Devries, BM, Clark, DD, Mellon, PL (2019) Differential CRE expression in Lhrh-cre and GnRH-cre alleles and the impact on fertility in Otx2-Flox mice. Neuro-endocrinology 108:328-342.

Hughes, AT, Croft, CL, Samuels, RE, Myung, J, Takumi, T, Piggins, HD (2015) Constant light enhances synchrony among circadian clock cells and promotes behavioral rhythms in VPAC2-signaling deficient mice. Sci Rep 5:14044.

Hughes, AT, Fahey, B, Cutler, DJ, Coogan, AN, Piggins, HD (2004) Aberrant gating of photic input to the suprachiasmatic circadian pacemaker of mice lacking the VPAC2 receptor. $J$ Neurosci 24:3522-3526.

Hughes, AT, Piggins, HD (2008) Behavioral responses of Vipr2-/- mice to light. J Biol Rhythms 23:211219.

Isobe, Y, Muramatsu, K (1995) Day-night differences in the contents of vasoactive intestinal peptide, gastrin-releasing peptide and Arg-vasopressin in the suprachiasmatic nucleus of rat pups during postnatal development. Neurosci Lett 188:45-48.

Jones, JR, Simon, T, Lones, L, Herzog, ED (2018) SCN VIP neurons are essential for normal lightmediated resetting of the circadian system. J Neurosci 38:7986-7995.

Lee, IT, Chang, AS, Manandhar, M, Shan, Y, Fan, J, Izumo, M, Ikeda, Y, Motoike, T, Dixon, S, Seinfeld, JE, et al. (2015) Neuromedin s-producing neurons act as essential pacemakers in the suprachiasmatic nucleus to couple clock neurons and dictate circadian rhythms. Neuron 85:1086-1102.

Liu, D, Stowie, A, de Zavalia, N, Leise, T, Pathak, SS, Drewes, LR, Davidson, AJ, Amir, S, Sonenberg, N, Cao, R (2018) mTOR signaling in VIP neurons regulates circadian clock synchrony and olfaction. Proc Natl Acad Sci USA 115:E3296-E3304.

Lucassen, EA, van Diepen, HC, Houben, T, Michel, S, Colwell, CS, Meijer, JH (2012) Role of vasoactive intestinal peptide in seasonal encoding by the suprachiasmatic nucleus clock. Eur J Neurosci 35:1466-1474.

Matthaei, KI (2007) Genetically manipulated mice: a powerful tool with unsuspected caveats. J Physiol 582:481-488.

Maywood, ES, Chesham, JE, O’Brien, JA, Hastings, MH (2011) A diversity of paracrine signals sustains molecular circadian cycling in suprachiasmatic nucleus circuits. Proc Natl Acad Sci USA 108:14306-14311.

Maywood, ES, Reddy, AB, Wong, GK, O’Neill, JS, O’Brien, JA, McMahon, DG, Harmar, AJ, Okamura, H, Hastings, $\mathrm{MH}$ (2006) Synchronization and maintenance of timekeeping in suprachiasmatic circadian clock cells by neuropeptidergic signaling. Curr Biol 16:599-605.

Mazuski, C, Abel, JH, Chen, SP, Hermanstyne, TO, Jones, JR, Simon, T, Doyle, FJ, Herzog, ED (2018) Entrainment of circadian rhythms depends on firing rates and neuropeptide release of VIP SCN neurons. Neuron 99:555-563.

Mei, L, Fan, Y, Lv, X, Welsh, DK, Zhan, C, Zhang, EE (2018) Long-term in vivo recording of circadian rhythms in brains of freely moving mice. Proc Natl Acad Sci USA 115:4276-4281. 
Meijer, JH, Michel, S, Vanderleest, HT, Rohling, JH (2010) Daily and seasonal adaptation of the circadian clock requires plasticity of the SCN neuronal network. Eur J Neurosci 32:2143-2151.

Miller, JE, Granados-Fuentes, D, Wang, T, Marpegan, L, Holy, TE, Herzog, ED (2014) Vasoactive intestinal polypeptide mediates circadian rhythms in mammalian olfactory bulb and olfaction. J Neurosci 34:6040-6046.

Moore, RY (2013) The suprachiasmatic nucleus and the circadian timing system. Prog Mol Biol Transl Sci 119:1-28.

Partch, CL, Green, CB, Takahashi, JS (2014) Molecular architecture of the mammalian circadian clock. Trends Cell Biol 24:90-99.

Power, A, Hughes, AT, Samuels, RE, Piggins, HD (2010) Rhythm-promoting actions of exercise in mice with deficient neuropeptide signaling. J Biol Rhythms 25:235-246.

Prosser, HM, Bradley, A, Chesham, JE, Ebling, FJ, Hastings, MH, Maywood, ES (2007) Prokineticin receptor 2 (Prokr2) is essential for the regulation of circadian behavior by the suprachiasmatic nuclei. Proc Natl Acad Sci USA 104:648-653.

Rezai Amin, S, Gruszczynski, C, Guiard, BP, Callebert, J, Launay, JM, Louis, F, Betancur, C, Vialou, V, Gautron, S (2019) Viral vector-mediated Cre recombinase expression in substantia nigra induces lesions of the nigrostriatal pathway associated with perturbations of dopaminerelated behaviors and hallmarks of programmed cell death. J Neurochem 150:330-340.

Taniguchi, H, He, M, Wu, P, Kim, S, Paik, R, Sugino, K, Kvitsiani, D, Fu, Y, Lu, J, Lin, Y, et al. (2011) A resource of Cre driver lines for genetic targeting of GABAergic neurons in cerebral cortex. Neuron 71:995-1013.

Todd, WD, Fenselau, H, Wang, JL, Zhang, R, Machado, NL, Venner, A, Broadhurst, RY, Kaur, S, Lynagh, T, Olson, DP, et al. (2018) A hypothalamic circuit for the circadian control of aggression. Nat Neurosci 21:717-724.

Varadarajan, S, Tajiri, M, Jain, R, Holt, R, Ahmed, Q, LeSauter, J, Silver, R (2018) Connectome of the suprachiasmatic nucleus: new evidence of the core-shell relationship. eNeuro 5(5):pii.

Viollet, C, Simon, A, Tolle, V, Labarthe, A, Grouselle, D, Loe-Mie, Y, Simonneau, M, Martel, G, Epelbaum, J (2017) Somatostatin-IRES-Cre mice: between knockout and wild-type? Front Endocrinol (Lausanne) 8:131.

Vosko, AM, Schroeder, A, Loh, DH, Colwell, CS (2007) Vasoactive intestinal peptide and the mammalian circadian system. Gen Comp Endocrinol 152:165-175.

Wen, S, Ma, D, Zhao, M, Xie, L, Wu, Q, Gou, L, Zhu, C, Fan, Y, Wang, H, Yan, J (2020) Spatiotemporal single-cell analysis of gene expression in the mouse suprachiasmatic nucleus. Nat Neurosci. 23(3):456-467.

Yoo, SH, Yamazaki, S, Lowrey, PL, Shimomura, K, Ko, CH, Buhr, ED, Siepka, SM, Hong, HK, Oh, WJ, Yoo, OJ, et al. (2004) PERIOD2::LUCIFERASE real-time reporting of circadian dynamics reveals persistent circadian oscillations in mouse peripheral tissues. Proc Natl Acad Sci USA 101:53395346.

Zhang, R, Lahens, NF, Ballance, HI, Hughes, ME, Hogenesch, JB (2014) A circadian gene expression atlas in mammals: implications for biology and medicine. Proc Natl Acad Sci USA 111:1621916224. 